\title{
Optimal Investment Strategy on Institutions
}

\author{
Yucong $\mathrm{Wu}$ \\ Department of Telecommunications Engineering, School of Electrical and Electronic Engineering, \\ North China Electric Power University, Beijing, 102206, China \\ 346591653@163.com
}

\begin{abstract}
Keywords: Classification Index Comprehensive Evaluation model; Principal Component Analysis method; Fractal Dimension method; Mahalanobis Distance-based TOPSIS Evaluation method; Discrete Time Investment Portfolio Optimization model; Markowitz Fund Allocation model; Efficiency Factor
\end{abstract}

\begin{abstract}
The donation from charitable foundations is playing an increasingly important role in the source of financial support for colleges and universities. How to generate an optimal investment strategy is a problem that every foundation concerns. In this paper, we construct a model to select institutions with high potential for effectively utilizing funding and high education performance. Then we determine the investment amount and the duration of each institution, which leads to the calculation of the estimated return.
\end{abstract}

To evaluate candidate schools, we find the official data from the U.S. National Center on Education Statistics, which maintains an extensive database of survey information on nearly all post-secondary colleges and universities in the United States. Then we categorize the indexes into eight metrics based on two aspects: institution features and student features. Using Classification Index Comprehensive Evaluation model based on Principal Component Analysis method, we get the rankings of candidate schools. However, the relevance of the metrics leads to deviation on the model results. Hence we propose an optimal model. First, we objectively obtain the relative materiality between the metrics by determining indexes weight using Fractal Dimension method. Second, we formulate the Mahalanobis Distance-based TOPSIS Evaluation method to work out the reasonable ranking by eliminating impacts due to correlation metrics using sample covariance matrix.

In order to get the investment amount and the duration of each institution, we generate a Discrete Time Investment Portfolio Optimization model setting the estimated return as the objective function based on the Markowitz Fund Allocation model. In the real life, the estimated return dropped greatly when the proportion of our investment to the institution total funding shows a consistently low level. Accordingly, our model introduces a concept of Efficiency Factor to optimize the model and reveals the potential institutions.

At last, we perform a sensitivity analysis on normalized parameter and index weight of factors affecting the estimated return of Fractal Dimension method. Result is almost not affected by the change of the factors. Strengths and weaknesses of our model are also discussed in the paper. The model can also be applied in the investments such as stocks.

\section{Introduction}


With the continuous development of society, higher education has stepped into a universal stage all over the world. To help evaluate educational performance of undergraduates attending colleges and universities in the United States, we set up an evaluation system and generate an optimal investment strategy on donating a total of $\$ 100,000,000$ (US100 million) to an appropriate group of schools per year, for five years. We break down the problem into several parts:

Rankings of the candidate institutions

Allocation strategy of funding

Time duration for the money provided to bring a strong positive impact on student performance

Classification of the estimated return on each school's investment

\section{Graded Index Comprehensive Evaluation model}

Evaluation index system for Institution Assessment. As for the foundation selection candidate institutions for donation, there are mainly two aspects that count: the potential for effectively utilizing funding and high education performance of the institution,which are concrete reflected by the data in sample form.To evaluate candidate schools,we categorize the indexes into eight metrics based on two aspects:institution features and student features. Institution features could be comprehensively assessed by four aspects:degree awarded status of all departments, whether the institution operates currently,predominant degree awarded status, whether the school is on Heightened Cash Monitoring by the Department of Education(HCM). Similarly, student features could be comprehensively assessed by seven aspects: admission scores,total enrollment, the retention rates ,average net price for institutions,students average loan debt,the completion rate,students income status after graduation .

\section{Evaluation Index System for Institution Assessment}
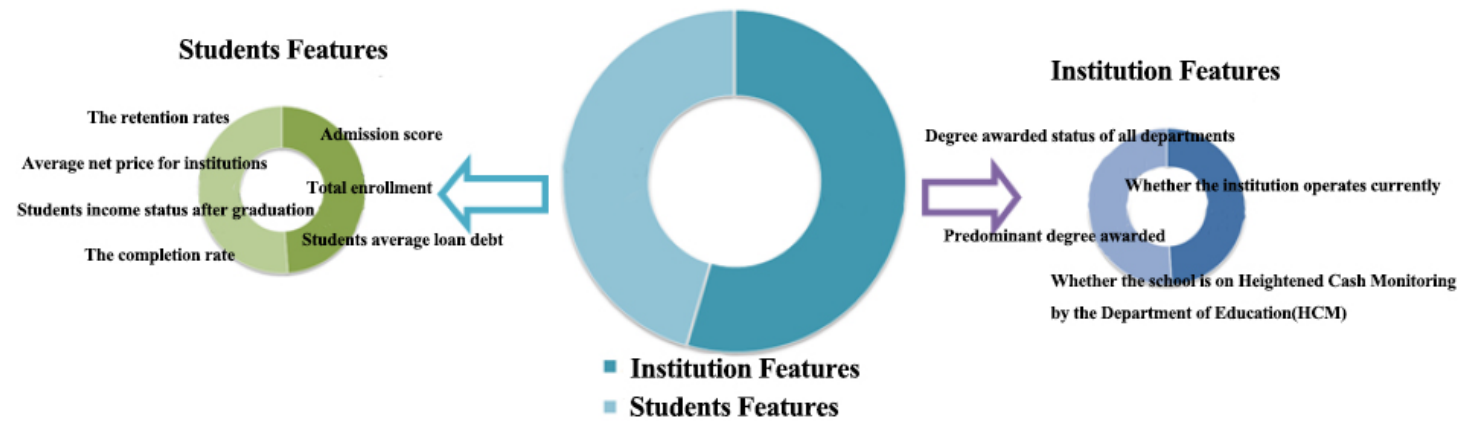

Data Processing. After doing dimensionless on the data, we calculate the numerical value of the indexes by multipling the data with its weight coefficient and add them up.

The results shows as follows (using four institutions as example):

Table 1 The Numerical Value of the Second Grade Indexes

\begin{tabular}{ccccc}
\hline University Indexes & $\begin{array}{c}\text { Alabama } \\
\text { A\&M } \\
\text { University }\end{array}$ & $\begin{array}{c}\text { University of } \\
\text { Alabama at } \\
\text { Birmingham }\end{array}$ & $\begin{array}{c}\text { University of } \\
\text { Alabama in } \\
\text { Huntsville }\end{array}$ & $\begin{array}{c}\text { Alabama } \\
\text { State } \\
\text { University }\end{array}$ \\
\hline Whether the institution operates currently & 1 & 1 & 1 & 1 \\
Predominant degree awarded status & 3 & 3 & 3 & 3 \\
Whether the school is on HCM & 0 & 0 & 0 & 0 \\
All departments degree awarded status & 0.29056 & 0.29438 & 0.31122 & 0.33762 \\
Total enrollment & 4051 & 11200 & 5525 & 5354 \\
The index mark of admission scores & 4.2204488 & 1.4832644 & 2.1281828 & -4.1457319 \\
The retention rates of the students & 0.5945898 & 0.6371398 & 0.6252898 & 0.5427898 \\
Average net price for institutions & 15743.405 & 16438.405 & 17795.905 & 15003.905 \\
The index mark of students average loan debt & 1.5730856 & 0.3970652 & 0.3757932 & 1.6607911 \\
The completion rate of the students & 0.4483033 & 0.5482191 & 0.5330499 & 0.4226852
\end{tabular}


Candidate School Ranking. Principal Component Analysis. We use Principal Component Analysis method to rank the candidate schools. Principal Component Estimate is raised by Massy in 1965, which is a biased estimate of regression coefficient parameters. The same as other biased estimates such as ridge estimate, is designed to overcome the least squares estimation's unstability when designing ill conditioned matrix. Principal Component Estimate transfers original regression independent variables to another set of variables, which is major constituent and select some major constituents with importance as the new independent variables. Here we dispose some independent variables without great impact on the results and do a dimensionality reduction. Afterwards,we estimate the parameters of the model built after the selection using the least squares estimation.At last,we transfer back to the previous model to get the estimation of the parameters.

However, the results shows that the institutions ranked by this model is not with high visibility.

Correlation Analysis. Principal Component Analysis is applied to the evaluation of the metrics with low correlation coefficient. We doubt that the error may caused by the correlation of the second grade indexes.

Hence, we analyze the correlation between the indexes and find that the indexes correlate between the institution features.

Because of this,we improved our algorithm to eliminate the effect on indexes correlation. First,we objectively obtain the relative materiality between the metrics by determining indexes weight using Fractal Dimension method. Second,we formulate the Mahalanobis Distance-based TOPSIS Evaluation method to worked out the reasonable ranking by eliminating impacts due to correlation metrics using sample covariance matrix.

Fractal Dimension method. Zipf formula is proposed by G. K. Zipf in 1949.The formula reveals a conclusion of the discontinuous phenomenonin spatial distribution:according to rank sequence,every category of discontinuous distribution event has definite correspondence on the content,quantity and scale.

Import the data into Matlab, and calculate the weights of the data as follows:

Table 2 Weight Coefficient of the Indexes

\begin{tabular}{ccc}
\hline Primary Index & Secondary Index & $\begin{array}{c}\text { Weight } \\
\text { Coefficient }\end{array}$ \\
\hline \multirow{2}{*}{ In terms of } & Whether the school is on HCM & 0.0640 \\
School & Predominant degree awarded status & 0.3036 \\
& Whether the institution operates currently & 0.1343 \\
& Degree awarded status of all departments & 0.0343 \\
\hline \multirow{2}{*}{ In terms of } & Admission scores & 0.0194 \\
Students & Total enrollment & 0.0084 \\
& The retention rates of the students & 0.0712 \\
& Average net price for institutions & 0.0302 \\
& Students average loan debt & 0.1522 \\
& The completion rate of the students & 0.1408 \\
& Students income status after graduation & 0.0417 \\
\hline
\end{tabular}

Table 2 shows that in the aspects of first grade index,institutions and students respectively weigh $0.5362,0.4638$. That is to say institutions are the first thing for the foundation to consider when doing the investment, which is rather common in the real life.

The Mahalanobis Distance-based TOPSIS Evaluation method. The Euclidean distance in traditional TOPSIS method can not tackle with the correlation between the indexes. Hence,it does 
double counting on the common information between the indexes. The more it correlates,the more it double counts.

Because of this,we use the Mahalanobis Distance to improve the traditional method named as the Mahalanobis Distance-based TOPSIS Evaluation method.We formulate the Mahalanobis Distance-based TOPSIS Evaluation method to worked out the reasonable ranking by eliminating impacts due to correlation metrics using sample covariance matrix.

Table 3 Rankings of Top 10 Candidate School

\begin{tabular}{ccc}
\hline Candidate School & $\begin{array}{c}\text { Comprehensive Score } \\
\text { (Neartude) }\end{array}$ & Ranking \\
\hline Williams College & 0.68275849 & 1 \\
Stanford University & 0.674260049 & 2 \\
Princeton University & 0.670833033 & 3 \\
Massachusetts Institute of Technology & 0.669036674 & 4 \\
Haverford College & 0.665358298 & 5 \\
University of Pennsylvania & 0.646217147 & 6 \\
Brown University & 0.644149199 & 7 \\
Georgetown University & 0.641504783 & 8 \\
Wellesley College & 0.636102676 & 9 \\
University of California, Berkeley & 0.610687599 & 10 \\
\hline
\end{tabular}

It shows that in Table 3, the top 10 candidate schools are with high prestige. Among them are world famous universities, it indicates that our algorithm and model are highly accurate and credible.

\section{Discrete Time Investment Portfolio Optimization model}

The initial formulation of the model. Discrete Time Investment Portfolio Optimization model is based on the Markowitz Fund Allocation model. The Markowitz Fund Allocation model is a solution to optimal portfolio investment stock. When the quantity of the shares increases, the estimation required basically is rather large, which causes unstabitily. Based on these problems, we mainly discuss mean variance portfoliooptimization with fixed transaction cost and proportional transaction cost in limited period, and obtain the analytic form solution to the problem. Besides, we give the solution to non traded regional boundary and efficient frontier.

In this model we set the estimated return as the objective function. The estimated return is directly related to output and input. Besides, output is estimated by the retention rates of the students, the completion rate of the students and students income status after graduation. Similarly, input is mainly estimated by the foundation investment.

Efficiency Factor. In the allocation of funds, we need to consider a problem, the amount of investment in the total amount of investment in a school to get the proportion of the total investment amount. If a school itself can get a lot of investment amount, then we even put a lot of money, it is difficult to bring the expected return. Therefore, we define the concept of efficiency factor.

Efficiency factor and students enrolled in grades, student income and owing on the loan, the main degree awarded the three factors related, and if these three factors are generally large, this school will be better and better, the total capital will be more and more, then we put the money brought yields and ideal situation compared to a lot of difference. So the efficiency factor and student enrollment, student income and owing on the loan, the main degree awarded the three factors into Anti correlation. So, we define: 


$$
E=0.4 * \frac{1}{U_{13}}+0.4 * \frac{1}{U_{21}}+0.2 * \frac{1}{U_{27}}
$$

Then the objective function needs to change, can be used to measure the yield. The equivalent yield is related to the yield and efficiency factor, and the yield should be less than the yield:

$R^{\prime}=R^{*} E$

$R^{\prime}$ is reduced rate of return. $F_{\text {is efficiency factor }}$

After introducing the efficiency factor, the solution model is obtained:

It is clear that the amount of money allocated in the table after the introduction of the efficiency factor has changed, so that the amount of money we invest will be much closer to what we expect.

Efficiency factor also has an impact on the rate of return, the rate of return of about 0.5 fluctuations, although a slight decrease, but this is a new equivalent return rate, more in line with the actual situation

\section{Conclusion}

It shows from the result of Graded Index Comprehensive Evaluation model that institutions such as Williams College and Stanford University are with high potential for effectively utilizing funding and high education performance, which reveal that these institutions are suitable for our investment.

The result of Discrete Time Investment Portfolio Optimization model is as follows.

Table 4 The Investment Strategy

\begin{tabular}{cccccc}
\hline Institutions & The First & The Second & The Third & The Forth & The Fifth \\
Year & Year & Year & Year & Year \\
\hline Williams College & 45.70 & 2665.42 & 469.93 & 0.00 & 393.09 \\
Stanford University & 207.87 & 0.00 & 1162.74 & 113.17 & 0.00 \\
Princeton University & 132.69 & 24.99 & 0.00 & 0.00 & 376.69 \\
MIT & 27.46 & 1.60 & 216.75 & 486.07 & 381.95 \\
Haverford College & 114.08 & 0.00 & 232.19 & 0.00 & 112.88 \\
University of Pennsylvania & 37.97 & 2.95 & 201.78 & 0.00 & 405.42 \\
Brown University & 54.13 & 2.07 & 155.35 & 42.63 & 403.83 \\
Georgetown University & 91.72 & 4.05 & 0.00 & 417.00 & 216.28 \\
Wellesley College & 187.18 & 595.89 & 1376.01 & 7.81 & 356.45 \\
University of California, Berkeley & 63.41 & 2.95 & 0.00 & 248.86 & 367.59 \\
Harvard University & 648.96 & 0.00 & 137.00 & 435.04 & 150.81 \\
University of Michigan, Ann Arbor & 79.34 & 0.00 & 0.00 & 350.37 & 173.92 \\
Reed College & 68.42 & 43.59 & 179.51 & 0.00 & 0.00 \\
Whitman College & 58.66 & 73.47 & 164.42 & 639.38 & 384.39 \\
Lafayette College & 30.83 & 1.09 & 977.63 & 338.63 & 186.84 \\
Smith College & 21.07 & 0.00 & 325.07 & 712.91 & 360.44 \\
Vanderbilt University & 49.82 & 5.27 & 0.00 & 168.69 & 373.84 \\
Carnegie Mellon University & 98.59 & 119.23 & 202.66 & 809.47 & 400.50 \\
Bates College & 24.21 & 0.00 & 0.00 & 0.00 & 100.28 \\
Santa Clara University & 106.01 & 1144.43 & 566.51 & 385.94 & 379.39 \\
University of Florida & 2005.84 & 834.34 & 0.00 & 539.18 & 352.10 \\
Drake University & 601.51 & 415.85 & 146.25 & 226.53 & 125.48 \\
Syracuse University & 34.33 & 282.73 & 0.00 & 140.61 & 395.05 \\
Saint Norbert College & 155.98 & 0.00 & 0.00 & 487.17 & 388.93 \\
Ohio Northern University & 41.75 & 0.90 & 232.51 & 0.00 & 364.14 \\
& & & & &
\end{tabular}




\begin{tabular}{cccccc} 
Elizabethtown College & 234.54 & 2041.86 & 166.52 & 188.75 & 370.81 \\
Pacific University & 122.92 & 0.00 & 156.07 & 292.97 & 0.00 \\
University of Kentucky & 73.72 & 0.00 & 0.00 & 652.39 & 0.00 \\
Marlboro College & 1297.43 & 0.00 & 0.00 & 286.99 & 140.39 \\
Biola University & 85.33 & 1541.25 & 681.86 & 722.03 & 402.20 \\
Gordon College & 170.28 & 0.00 & 273.09 & 92.00 & 398.75 \\
Bethel University & 889.53 & 0.00 & 177.70 & 0.00 & 396.94 \\
La Salle University & 143.60 & 0.00 & 818.14 & 0.00 & 0.00 \\
San Jose State University & 336.41 & 0.00 & 151.27 & 561.19 & 0.00 \\
Messiah College & 496.70 & 0.00 & 189.70 & 594.55 & 200.89 \\
Michigan Technological University & 272.13 & 2.20 & 390.09 & 0.00 & 386.71 \\
University of the Pacific & 457.11 & 186.65 & 249.25 & 0.00 & 161.95 \\
King's College & 432.75 & 7.21 & 0.00 & 59.64 & 391.06 \\
\hline Rate of Return & 0.527994 & 0.467454 & 0.504444 & 0.478307 & 0.492884 \\
\hline
\end{tabular}

We donate intensively in the first stage, and scattered afterwards. Not all the institutions we donate are the most famous universities, among them are also institutions with high potential.

Hence,according to the two model,we can effectively utilize the 100 million to donate the candidate school with the optimal strategy.

\section{Reference}

[1] Jijian Wang. Research on the Questions of National College Students' Mathematical Modeling Contest [M] . Beijing: National Defense Industry Press, 2005. 250.

[2] Jisheng Liu, Yanguang Chen. Fractal Dimension Method of Urban System Rank Structure and Its Measuring Method [J]. Geographical Research, 1998, 17(1): 82-89.

[3] Zhengxin Wang. TOPSIS Decision Making Method Based on Markov Distance and Its Application [J]. Economic Mathematic, 2012, 29(2): 18-20.

[4] Fogel D B, “An introduction to simulated evolutionary optimization,” IEEE Transaction Neural Network, vol.5, no.1, pp.3-14, 1994.

[5] Huixuan Gao, Applied Multivariate Statistical Analysis, Beijing: Peking University Press, 2006.

[6] Markowitz H.Portfolio selection. The Journal of Finance. 1952, 7(1): 77-91.

[7] Tobin J. Liquidity Preference as Behavior towards Risk. Review of Economic

Studies. 1958, 25(2), 65-86.

[8] Markowitz H. Portfolio Selection: Efficient Diversification of Investments. John Wiley \& Sons, 1958.

[9] Sharpe W. F.A Simplified Model for Portfolio Analysis. Management Science. 1963, 9(3), 277-293.

[10] Sharp W.F. Capital Asset prices: A Theory of Market Equilibrium Under Conditions of Risk. The Journal of Finance. 1964, 19(3), 425-442.

[11] Lintner J.The Valuation of Risk Assets and the Selection of Risky Investment in Stock Portfolio and Capital Budgets. The Review of Economic and Statistics. 1965, 47(1), 13-37.

[12] Simaan Y. Estimation Risk in Portfolio Selection: the Mean Variance Model Versus the Mean

Absolute Deviation Model. Management Science. 1997, 43(10), 1437-1446.

[13] Mansini R., Speranza M. G. Heuristic algorithms for the portfolio selection problem with minimum transaction lots. European Journal of Operational Research. 1999, 114(2), 219-233.

[14] Speranza M.G.Linear programming models for portfolio optimization. Finance. 1993, 14, 107-123. 